\title{
Risikobewertung und lebensmittelrechtliche Beurteilung von Fisch- und Fischerzeugnissen aus mit marinen Mykobakterien infizierten Aquakulturbeständen
}

\author{
Dr. H. Göhner, G. Tardel und Prof. Dr. Dr. F. Feldhusen \\ Landesamt für Landwirtschaft, Lebensmittelsicherheit und Fischerei, Mecklenburg-Vorpommern
}

\section{Zusätzliche Informationen/Anlagen}

1. Stellungnahme des BfR (FLI, BfEL) - Anfrage Schleswig-Holstein - AZ 5282-02-2784387 Gesundheitliche Gefährdung Fischereierzeugnisse/Fischtubekulose-05.01.2006

2. Risikobewertung des BfR (FLI, BfEL) - Anfrage MecklenburgVorpommern - AZ 5282-02-2849924; Lebensmittelrechtliche Beurteilung für Nachweis Mycobacterium marinum beim Stör - 17.03.2006

\section{Zusammenfassung/Erläuterung}

Im Jahr 2005 gab es in zwei Bundesländern Nachweise mariner Mykobakterien bei Fischen sowie deren Erzeugnissen aus Aquakulturanlagen.

Moribunde Fische zeigten hier selten das charakteristische Bild einer chronisch-granulomatösen Mykobakteriose, sondern erkrankten atypisch unter septischem Verlauf mit Erregernachweis aus sämtlichen inneren Organen.

Da auch eine oral-alimentäre Übertragung dieser Mykobakterien auf den Menschen durch kontaminierte Fische oder
Fischerzeugnisse (z. B. Kaviar) nicht auszuschließen ist, besteht aufgrund einer Bewertung des BfR (2006) ein geringes bzw. z.Z. nicht abschließend einzuschätzendes gesundheitliches Risiko für den Verbraucher, wenn derartige Produkte als Lebensmittel in den Verkehr gebracht werden. Dies gilt insbesondere, wenn Zubereitungsmethoden nicht zur Abtötung des Erregers führen.

Dargestellt werden die wichtigsten Aussagen der Risikobewertung des BfR. Die sich daraus ergebenden lebensmittelrechtlichen Fragestellungen sollen diskutiert werden: (a) Vorgehen der amtlichen Lebensmittel- und Tierseuchenüberwachung, (b) Bewertung von Fischen als Lebensmittel mit unterschiedlichen Befunden aus einem infizierten Bestand (gesunde; klinisch kranke mit oder ohne chronische Organveränderungen) und (c) lebensmittelrechtliche Beurteilung der amtlich untersuchten, erregerhaltigen, im Verkehr befindlichen Einzelprobe.

\section{Beschluss}

Kenntnisnahme 